\title{
Terapia alternativa para microvarizes e telangiectasias com uso de agulha
}

\author{
Alternative therapy for microvarices and telangiectasias \\ with use of needle \\ Raimundo Rosendo de Oliveira ${ }^{1}$, Eraldo Barbosa Calado ${ }^{1}$, Diógenes Luis da Mota ${ }^{2}$,
André Filipe Vieira Pereira da Silva ${ }^{3}$, Jennecy Sales Cavalcanti ${ }^{4}$
}

\section{Resumo}

Contexto: $\mathrm{O}$ desenvolvimento de terapia alternativa à convencional para a destruição de microvarizes e telangiectasias sem o uso de produtos químicos tem como objetivo reduzir os efeitos colaterais, faz uso de agulha para lise mecânica dos vasos e tem como modelo experimental galinhas da linhagem Lohmann Brown.

Objetivo: Elaborar uma nova técnica, desenvolvendo um tratamento alternativo, sem uso de produtos químicos, objetivando a redução dos efeitos colaterais.

Métodos: Foram utilizadas 30 galinhas da linhagem Lohmann Brown, sendo que 15 foram submetidas ao método convencional de tratamento de microvarizes e telangiectasias (grupo-controle) e as outras 15 receberam o tratamento experimental proposto (grupo experimental). O grupo experimental foi tratado com agulha de lise vascular, percorrendo todo o trajeto dos vasos escolhidos em punções escalonadas até que todo o vaso ser atingido. $\mathrm{O}$ grupo-controle foi tratado com oleato de monoetanolamina e glicose a $50 \%$, puncionando-se o vaso com agulha $13 \times 3 \mathrm{~mm}$ e injetando-se, em média, $0,3 \mathrm{~mL}$ da solução em cada vaso.

Resultados: Dos 50 vasos tratados no grupo experimental, dois apresentaram recidiva total, cinco apresentaram recidiva parcial, e 43 apresentaram destruição (lise) satisfatória; enquanto que, no grupo-controle, dos 51 vasos tratados, quatro apresentaram recidiva total, 12 , recidiva parcial, 22, destruição satisfatória, e em 13 ocorreu endurecimento de trajeto.

Conclusão: O presente estudo demonstrou que o método experimental proposto, com uso de agulha de lise vascular, possui mais eficiência no tratamento de microvarizes se comparado com o método convencional, devido à redução das recidivas e à ausência de hipercromia de trajeto endurecido.

Palavras-chave: Vascular, tratamento, microvarizes.

\begin{abstract}
Background: The development of an alternative to the conventional therapy for microvarices and telangiectasias without using chemical products aims at reducing side effects, using a needle for mechanical lysis of vessels. It uses Lohmann Brown hens as the experimental model.

Objective: To develop a new technique, creating an alternative treatment without using chemical products, aiming at the reduction of side effects.

Methods: Thirty Lohmann Brown hens were used, 15 submitted to the conventional method of treating microvarices and telangiectasias (control group) and 15 receiving the proposed experimental treatment (experimental group). The experimental group was treated using a needle for vascular lysis, passing through the whole extension of chosen vessels in staggered punctures until the whole vessel was reached. The control group was treated with ethanolamine oleate and $50 \%$ glucose, puncturing the vessel with a $13 \times 3 \mathrm{~mm}$ needle and injecting on average $0.3 \mathrm{~mL}$ of the solution in each vessel.

Results: Of the 50 vessels treated in the experimental group, two presented total recurrence, five partial recurrence and 43 satisfactory destruction (lysis), whereas of the 51 vessels treated in the control group, four presented total recurrence, 12 partial recurrence, 22 satisfactory destruction and 13 presented conduit vessel stiffness.

Conclusion: The present study demonstrated that the experimental method using a needle for vascular lysis is more efficient for the treatment of microvarices when compared with the conventional method, due to reduction in recurrence rates and absence of hyperchromatism of conduit vessel stiffness.
\end{abstract}

Keywords: Treatment, vascular, microvarices.

1. Professor, Universidade do Estado do Rio Grande do Norte (UERN), Mossoró, RN.

2. Professor, Programa de Pós-Graduação, Departamento de Patologia, Universidade Federal de Pernambuco (UFPE), Recife, PE. Pesquisador, Laboratório de Citologia Clínica e Histologia Quantitativa, Departamento de Ciências Farmacêuticas, UFPE, Recife, PE.

3. Pesquisador, Laboratório de Citologia Clínica e Histologia Quantitativa, Departamento de Ciências Farmacêuticas, UFPE, Recife, PE. Professor, Faculdade Maurício de Nassau, Recife, PE.

4. Professor, Programa de Pós-Graduação, Departamento de Patologia, UFPE, Recife, PE.

Artigo submetido em 29.08.06, aceito em 21.02.07.

J Vasc Bras 2007;6(1):17-24.

Copyright $\odot 2007$ by Sociedade Brasileira de Angiologia e de Cirurgia Vascular. 


\section{Introdução}

As varizes constituem uma das doenças mais antigas de que se tem relato e, atualmente, estão presentes, em média, em torno de 30 a $40 \%$ da população brasileira. Alguns fatores de riscos são considerados, tais como ocupação, gravidez, dieta, obesidade, hereditariedade e etnia, sendo as mulheres as mais afetadas, numa razão de $4: 1$. Acredita-se que $70 \%$ das mulheres com idade acima de 40 anos apresentem veias varicosas ${ }^{1-7}$.

Existem dois tipos de varizes: as chamadas primárias, que aparecem influenciadas pela tendência hereditária, e as chamadas secundárias, que aparecem por doenças adquiridas no decorrer da vida, sendo de tratamento mais difícil. As varizes primárias são as responsáveis pelas antiestéticas linhas vermelhas e azuis, de diversos tamanhos, que aparecem especialmente nas pernas das mulheres e também pelas varizes de maior calibre, que são as mais freqüentes. As varizes secundárias são causadas por uma doença pré-existente, como fístulas arteriovenosas (congênitas ou adquiridas), traumas, angiodisplasias, síndrome pós-flebítica, compressões extrínsecas, etc. ${ }^{8}$

As principais teorias sobre etiologia das varizes primárias ou essenciais dos membros inferiores estão relacionadas com alterações na parede da veia, com modificação na estrutura do colágeno e/ou elastina, aumentando a presença de material elástico com o espessamento do vaso $^{9}$, incompetência valvar localizada ou segmentar e presença de fístulas arteriovenosas no nível da microcirculação. As varizes secundárias estão relacionadas com a síndrome pós-flebítica, gravidez, fístulas arteriovenosas traumáticas, angiodisplasias e compressões extrínsecas ${ }^{10}$.

Com os avanços no conhecimento da doença venosa crônica, houve necessidade de se expandir as definições que pudessem abranger muitos de seus aspectos, incluindo os anatômicos, fisiopatológicos e etiológicos. Com essa finalidade, foi elaborado, em 1994, pelo Comitê Internacional do Fórum Venoso Americano, um documento de consenso para a classificação da doença venosa (Tabela 1), que se baseava no quadro clínico, nos fatores etiológicos, na distribuição anatômica e nos achados fisiopatológicos, denominado de classificação clínica, etiológica, anatômica e fisiopatológica (CEAP) $)^{11-16}$

Alguns autores ${ }^{17,18}$ consideram a classificação CEAP bastante trabalhosa quando utilizada na sua forma completa, não sendo utilizada na prática do atendimento de pacientes.

No seu estudo, Maffei ${ }^{19}$ encontrou uma prevalência de $82 \%$ de telangiectasia, o que o levou a considerá-la como uma característica da raça humana, passando a constituir-se uma doença quando se torna muito extensa, representando, dessa forma, um risco para o portador que se queixa de dor ou queimação na região ${ }^{20}$, especialmente mulheres no período menstrual.

As telangiectasias são pequenos capilares localizados na pele, muito finos, ramificados, em geral de coloração avermelhada, constituídos de microfístulas arteriovenosas. São também chamadas spider veins, pelo padrão tipo teias de aranha, e, na grande maioria das vezes, causam distúrbios apenas do ponto de vista estético $^{21-22}$. Podemos afirmar que as telangiectasias são definidas como dilatações intradérmicas das veias, cujo diâmetro estimado é de aproximadamente $1 \mathrm{~mm}^{22-23}$.

$\mathrm{Na}$ terapêutica clínica das varizes, tornam-se necessárias medidas gerais como: combater o excesso de peso corporal, estimular a atividade física, evitar o ortostatismo prolongado e o uso de fármacos como estrogênio/ progesterona, tireotrófico e bloqueadores do canal de cálcio ${ }^{24}$.

A escleroterapia, conhecida como "aplicação", é, no momento, o tratamento de escolha destinado às pessoas acometidas de telangiectasias, sendo acompanhada por um conjunto de medidas, desde dieta até exercícios físicos orientados, bem como uso de meias elásticas, controle hormonal e suspensão do uso de anticoncepcionais $^{25,26}$.

A finalidade do tratamento é a oclusão do tronco varicoso em questão. A idéia é introduzir na veia uma substância irritante, que induza um processo inflamató- 
rio, levando à fibrose, fazendo com que o vaso perca seu caráter cilíndrico e excluindo-o do caminho da circulação ${ }^{27}$.

A vantagem do tratamento com escleroterapia é que pode ser feito em sessões no consultório médico, e o paciente pode exercer suas atividades normais em poucos dias, mas seus efeitos colaterais ainda são um desafio para os angiologistas, dentre os quais destacamse: coágulos dentro de veias tratadas, hipercromia, úlcera química, dor persistente, dispnéia e urticária.

Os tratamentos realizados para os casos de telangiectasias, por mais desenvolvidos que já estejam, ainda requerem melhores resultados. Os principais desconfortos são: manchas hipercrômicas, pequenas queimaduras, recidivas e, principalmente, as dores causadas pelos distintos tratamentos utilizados na prática médica.

Para que o tratamento das telangiectasias aproxime-se do ideal, deve ter seu tempo de duração ainda menor, não deixar mancha e ser, acima de tudo, indolor. O presente estudo pretende elaborar uma nova técnica que se aproxime do ideal, eliminando ao máximo os desconfortos.

O presente trabalho pretendeu desenvolver tratamento alternativo, sem uso de produtos químicos, objetivando a redução dos efeitos colaterais. Procurou propor um tratamento para microvarizes e telangiectasias que não utilize produtos químicos; não gere recidivas; seja de rápida e fácil aplicação; não cause manchas; e apresente resultado imediato.

\section{Métodos}

\section{Considerações bioéticas}

O projeto para realização desse estudo recebeu parecer favorável do Comitê de Ética em Experimentação Animal da Universidade Federal de Pernambuco, através do Ofício $\mathrm{n}^{\circ} 58 / 05$, de acordo com as normas

Tabela 1 - Classificação clínica, etiológica, anatômica e fisiopatológica

\begin{tabular}{ll}
\hline Classe & Classificação clínica \\
\hline 0 & Nenhum sinal visível ou palpável de doença venosa \\
1 & Veias reticulares ou telangiectasia \\
2 & Veias varicosas \\
3 & Edema \\
4 & Alterações da pele (pigmentação, eczema venoso, lipodermatoesclerose) \\
5 & Alterações da pele, além de ulcerações cicatrizadas na pele, além de ulcerações ativas, em andamento \\
\hline
\end{tabular}


vigentes no Brasil, especialmente a Lei $\mathrm{n}^{\circ} 9.605$, artigo 32, e o Decreto ${ }^{\circ} 3.179$, artigo 17, de 21/09/1999, que trata da questão do uso de animais para fins científicos.

\section{Unidade experimental}

Foram utilizadas 30 galinhas da linhagem Lohmann Brown, sendo que 15 foram submetidas ao método convencional de tratamento de microvarizes e telangiectasias (denominado grupo-controle-GC), e as outras 15 receberam o tratamento experimental proposto (denominado grupo experimental - GE).

Seu crescimento foi acompanhado no biotério localizado na Universidade Estadual do Rio Grande do Norte, onde foram alimentadas com dietas específicas, industrializadas e suplementadas de acordo com a idade, sendo periodicamente pesadas em balança de precisão.

O biotério possui controle sanitário de manutenção, efetuado com pulverizações semanais à base de amoníaco, as vermifugações. $\mathrm{O}$ controle do ambiente foi feito por nebulizações, visando à melhora da umidade relativa do ar em períodos secos. Os animais foram acondicionados suspensos em gaiolas de arame galvanizado e suporte de ferro a $1,20 \mathrm{~cm}$ do chão e área de $25 \times 40 \mathrm{~cm}$, totalizando $1.000 \mathrm{~cm}^{2}$.

\section{Preparação do material}

Foi utilizado um motor de $12 \mathrm{v}$ para acoplagem da agulha, conectado a um transformador de $220 \mathrm{~V} / 12 \mathrm{~V}$, e um sistema de pedal para acionar o mecanismo que trabalha a uma velocidade de aproximadamente 240 rpm. O mecanismo possui uma haste que, ao ser acionada, executa movimentos no ângulo de $30^{\circ}$, promovendo à agulha acoplada um movimento longitudinal, de avanço e recuo. As agulhas de lise vascular são de fabricação artesanal, em aço inoxidável de $7 \mathrm{~mm}$ de espessura, possuindo triplo bisel, atingindo o epitélio e destruindo o vaso objetivado. Para acomodação do material, utilizou-se mesa auxiliar tipo Mayo coberta em campo estéril.

\section{Desenho do estudo}

Para realização do procedimento de destruição do vaso, as galinhas foram transportadas do biotério para o Hospital Veterinário, sendo duas em cada gaiola.
Foi feita a contenção dos pés do animal com auxílio de cadarços de algodão. Deitou-se o animal sobre a mesa, escolhendo-se, convencionalmente, o lado esquerdo de todos os animais. No campo cirúrgico onde seria realizado o experimento, foram retiradas penas da galinha suficientes para ampliação da área onde se encontravam os vasos. O campo estudado (região peitoral esquerda) foi registrado em máquina fotográfica digital para posterior comparação. A região estudada foi esterilizada com solução antisséptica (álcool iodado a $2 \%$ ); posteriormente, foi administrado, via intramuscular, o anestésico cetamina, na dosagem de $40 \mathrm{mg} / \mathrm{kg}$. Após cerca de 4 minutos, até que o animal apresente apresentasse sinais de estar totalmente anestesiado, iniciava-se o tratamento experimental.

O GE foi tratado com agulha de lise venosa, percorrendo todo o trajeto dos vasos escolhidos em punções escalonadas até que todo o vaso fosse atingido. O GC foi tratado com oleato de monoetanolamina e glicose a $50 \%$, puncionando-se o vaso com agulha $13 \times 3 \mathrm{~mm}$ e injetando-se, em média, $0,3 \mathrm{~mL}$ da solução em cada vaso. Imediatamente após o término do tratamento, (classificado como dia zero), a área foi novamente fotografada e resfriada com compressas de gelo. Também foram realizadas fotografias no sétimo e no $13^{\circ}$ dia após o tratamento.

Após 13 dias da destruição do vaso, tanto no GC como no GE, foi retirado tecido para a biópsia, tomando-se como base o local do halo cicatricial na projeção do vaso tratado. O material biopsiado foi fixado imediatamente após a retirada do corpo da galinha, para prevenir as alterações post-mortem, em Bouin, na proporção de 1:20. O tecido fixado foi posteriormente desidratado, diafanizado e parafinizado para formação do bloco, sendo este cortado no micrótomo com lâmina de 4 micrômetros de precisão.

O tecido cortado foi posicionado na lâmina, desparafinizado, hidratado, colocado em solução de Bouin, em estufa, a $56^{\circ} \mathrm{C}$ por 1 hora. Em seguida, foi lavado em água corrente até desaparecer a cor amarela dos cortes.

O tecido foi corado em solução de hematoxilinaeosina (HE) por 10 minutos e, em seguida, lavado em 
água corrente. Após as lâminas terem sido colocadas em água acética a $0,5 \%$ e mergulhadas em água destilada, foram desidratadas em álcool e xilol. Os resultados dos tratamentos foram comparados entre si a partir das lâminas histológicas que foram analisadas no microscópio óptico Zeiss, modelo Axioscope, utilizando-se as objetivas de $\mathrm{x} 4, \mathrm{x} 10 \mathrm{e} \mathrm{x} 40$. Esses animais puderam ser utilizados para outras atividades.

\section{Estatística}

Foi feita a comparação entre o número de vasos tratados e as ocorrências observadas. $\mathrm{O}$ teste utilizado foi o qui-quadrado de Fisher, e a significância do teste foi de $95 \%(\mathrm{p}<0,05)$. O software utilizado para o tratamento estatístico foi o Epi-Info, versão 6.0.4 $4^{28,29}$.

\section{Resultados}

Dos 15 animais tratados no GE, oito tiveram quatro vasos tratados cada (totalizando 32 vasos), quatro tiveram três vasos tratados cada (totalizando 12 vasos), e três tiveram dois vasos tratados cada (totalizando 6 vasos), constituindo um todo de 50 vasos tratados para esse grupo. Já no GC, dentre os 15 animais, oito tiveram quatro vasos tratados (totalizando 32 vasos), cinco tiveram três vasos tratados (totalizando 15 vasos), e dois tiveram dois vasos tratados (totalizando quatro vasos), constituindo um todo de 51 vasos tratado no GC.

Dos 50 vasos tratados no GE, dois apresentaram recidiva total, cinco apresentaram recidiva parcial, e 43 apresentaram destruição (lise) satisfatória; enquanto que, no GC, dos 51 vasos tratados, quatro apresentaram recidiva total, 12 , recidiva parcial, 22 , destruição satisfatória, e em 13 ocorreu endurecimento de trajeto (Figura 1).

Observa-se que, no GE, a ocorrência de destruição satisfatória foi mais freqüente, tendo menor freqüência nos casos de recidiva total, recidiva parcial e endurecimento do trajeto, onde não houve nenhum episódio. Comparando as freqüências entre grupos, houve diferença estatisticamente significante $(p=0,00003)$.

\section{Análise macroscópica}

Foi realizada a análise macroscópica das 30 galinhas da linhagem Lohmann Brown, sendo 15 tratadas com oleato de monoetanolamina e 15 tratadas com o método experimental, com uso de agulha de lise vascular nos dias zero, 7 e 13.

As galinhas do GE apresentaram equimose da região tratada, com duração média de 16 dias (três animais, 18 dias; nove animais, 16 dias; e três animais, 14 dias). Nos vasos em que ocorreu destruição satisfatória, a fibrose cicatricial do trajeto tratado permaneceu visível por 26 dias em média. No GC, apresentaram equimose da região tratada, com duração média de 21,2 dias, sendo 11 em 22 dias, três em 20 dias e um em 16 dias. Nos vasos em que ocorreu destruição satisfatória, a fibrose cicatricial do trajeto tratado permaneceu visível por 31 dias em média.

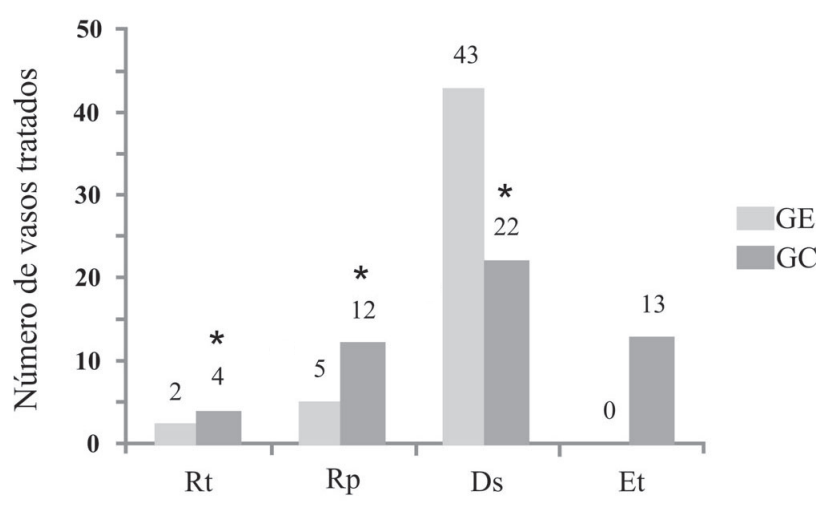

Ocorrência observada

$* \mathrm{p}<0,001$

$\mathrm{Ds}=$ destruição satisfatória; $\mathrm{Et}=$ endurecimento do trajeto; $\mathrm{GC}=$ grupocontrole; $\mathrm{GE}=$ grupo experimental; $\mathrm{Rp}=$ recidiva parcial; $\mathrm{Rt}=$ recidiva total;

Figura 1 - Ocorrência observada nos vasos dos grupos experimental e controle

\section{Análise histológica}

Nas preparações coradas com HE, observou-se, no GE, áreas de reparação tecidual e reepitelização com material fibrinóide na superfície. Na derme, observa-se infiltrado de células inflamatórias, presença de células mononucleadas (macrófagos) e sinais sugestivos de degeneração de pequenos vasos sangüíneos, os quais exibem estrutura histológica modificada. Esses aspectos não foram visualizados no $\mathrm{GC}$, onde o vaso perma- 
neceu íntegro, porém colabado e, eventualmente, com presença de hemácias aprisionadas.

\section{Discussão}

Pacientes que possuem microvarizes e que são submetidos a tratamentos convencionais, quer seja escleroterapia ou tratamento a laser, estão, muitas vezes, sujeitos a complicações diversas ${ }^{30}$, tais como hipercromia de trajeto tratado, alergia, necrose cutânea e recidiva do vaso previamente tratado, levando, com freqüência, a um resultado estético desagradável ${ }^{31}$. Dentre as técnicas convencionais mais utilizadas na prática clínica, estão as que fazem uso do oleato de monoetanolamina, aqui comparadas com o método experimental proposto, baseado em destruição mecânica com uso de agulha para lise vascular.

O objetivo dos métodos convencionais é levar a uma flebite química, obliteração do vaso e posterior absorção pelo organismo ${ }^{32}$. O método proposto foi idealizado com a finalidade de destruir diretamente o vaso, sem passar pelos estágios de flebite e obliteração, facilitando, assim, a absorção do mesmo e minimizando os efeitos colaterais.

O presente estudo demonstrou que o método experimental proposto, com uso de agulha para lise vascular, possui mais eficiência no tratamento das microvarizes, reduzindo as recidivas e não provocando manchas hipercrômicas.

O tratamento realizado por destruição mecânica através de agulha promove a lise imediata de vasos venosos e extravasamento das hemácias, não permitindo, com isso, que as hemácias desoxigenadas, aprisionadas dentro do vaso, liberem suas moléculas de ferro, levando, assim, à formação de manchas hipercrômicas de difícil tratamento e resultados estéticos indesejáveis $^{33}$.

No caso de recidivas, mesmo tendo ocorrido no tratamento com o GE, os valores foram significativamente inferiores aos observados no GC, devido à destruição mecânica provocar uma ação de lise direta sobre o vaso. As recidivas ocorridas no GE devem-se, provavelmente, à falha técnica em se percorrer o trajeto completo do vaso com a agulha de lise vascular, assim como à profundidade do vaso em relação à epiderme.

A avaliação da dor $^{34}$ requer experimentos mais elaborados, envolvendo a análise de mecanismos neurossensoriais, pois os animais estudados foram submetidos a efeitos de anestésicos gerais. Com uso de anestésicos tópicos no trajeto do vaso a ser tratado, poderíamos destruir o vaso sem que precisássemos de anestesia geral, porém isso não ocorre no caso do tratamento convencional, pois o uso de tal artifício leva, normalmente, à vasoconstricção ${ }^{19,20}$ e dificuldade na punção para injeção do esclerosante ${ }^{34}$.

Relatos de experimento semelhantes ao do presente estudo não foram encontrados na literatura científica. Para que possa ocorrer uma futura utilização do tratamento proposto, faz-se necessária a realização de experimentos em diversas situações, nos mais diferentes grupos de risco epidemiológico ${ }^{1,5,35}$ e em diferentes variações fisiológicas ${ }^{36,37}$, que podem ser observadas através de um estudo comparado em modelos animais diferentes. Com isso, é possível estabelecer diretrizes ${ }^{32}$ para a aplicação desse método em humanos. Até mesmo a combinação de técnicas ${ }^{38}$ pode ser a melhor solução para o tratamento de varizes.

\section{Conclusão}

O método experimental proposto, com uso de agulha para lise venosa, possui mais eficiência no tratamento das microvarizes, reduzindo as recidivas, não provocando hipercromia do trajeto tratado e mostrando-se mais eficiente a respeito da minimização dos transtornos estéticos, freqüentemente observados na técnica convencional com oleato de monoetanolamina a $5 \%$.

Para a aplicação clínica do método proposto, deve-se realizar experimentos que avaliem diferentes situações fisiológicas, observadas nos mais diferentes grupos de riscos. A técnica é de fácil aplicação, e os materiais são de fácil obtenção e baixo custo.

Como as etapas de flebite química e obliteração dos vasos foram abolidas desse experimento, o tratamento se dá com mais rapidez e menos efeitos colaterais em relação ao tratamento convencional. O sangue extrava- 
sado do vaso, após a lise mecânica, é completamente absorvido, não deixando manchas na pele.

\section{Referências}

1. Beebe-Dimmer JL, Pfeifer JR, Engle JS, Schottenfeld D. The epidemiology of chronic venous insufficiency and varicose veins. Ann Epidemiol. 2005;15:175-84.

2. Cleave TL. Varicose veins, nature's error or man's? Some implications of the Darwinian theory. Lancet. 1959;2:172-5.

3. Cornu-Thenard A, Boivin P, Baud JM, De-Vincenzi I, Carpentier PH. Importance of the familial factor in varicose disease. Clinical study of 134 families. J Dermatol Surg Oncol. 1994;20:318-26.

4. Evans CJ, Fowkes FG, Ruckley CV, Lee AJ. Prevalence of varicose veins and chronic venous insufficiency in men and women in the general population: Edinburgh Vein Study. J Epidemiol Community Health. 1999;53:149-53.

5. Iannuzzi A, Panico S, Ciardullo AV, et al. Varicose veins of the lower limbs and venous capacitance in postmenopausal women: relationship with obesity. J Vasc Surg. 2002;36:965-8.

6. Maffei FH, Magaldi C, Pinho SZ, et al. Varicose veins and chronic venous insufficiency in Brazil: prevalence among 1755 inhabitants of a country town. Int $\mathrm{J}$ Epidemiol. 1986;15:210-7.

7. Sadick NS. Predisposing factors of varicose and telangiectatic leg veins. J Dermatol Surg Oncol. 1992;18:883-6.

8. London NJ, Nash R. ABC of arterial and venous disease. Varicose veins. BMJ. 2000;320:1391-4.

9. Azizi MAA. Morfometria das fibras elásticas em colaterais varicosas do sistema de veias safenas [dissertação]. Rio de Janeiro: UERJ; 2005.

10. Johnson Jr. G, Rutherford RB. Varicose veins: patients selection and treatment. In: Rutherford RB, editor. Vascular surgery. 5th ed. Phliladelphia: WB Saunders; 2000. p. 2033.

11. Beebe HG, Bergan JJ, Bergqvist D, et al. Classification and grading of chronic venous disease in the lower limbs. Aconsensus statement. Eur J Vasc Endovasc Surg. 1996;12:487-91; discussion 491-2.

12. Kistner RL, Eklof B, Masuda EM. Diagnosis of chronic venous disease of the lower extremities: the "CEAP" classification. Mayo Clin Proc. 1996;71:338-45.

13. Kistner RL. Definitive diagnosis and definitive treatment in chronic venous disease: a concept whose time has come. J Vasc Surg. 1996;24:703-10.

14. Labropoulos N, Giannoukas AD, Delis K, et al. Where does venous reflux start? J Vasc Surg. 1997;26:736-42.
15. Porter JM, Moneta GL. Reporting standards in venous disease: an update. International Consensus Committee on Chronic Venous Disease. J Vasc Surg. 1995;21:635-45.

16. Vin F. Varices, varicose veins. EMC Cardiologie Angéiologie. 2005;2:1-26.

17. Rutherford RB. Vascular surgery--comparing outcomes. J Vasc Surg. 1996;23:5-17.

18. Rutherford RB, Padberg FT Jr., Comerota AJ, Kistner RL, Meissner MH, Moneta GL. Venous severity scoring: an adjunct to venous outcome assessment. J Vasc Surg. 2000;31:1307-12.

19. Maffei FHA. Contribuição para o conhecimento da epidemiologia das varizes e da insuficiência venosa crônica dos membros inferiores [tese]. Botucatu: UNESP; 1982.

20. Maffei FHA, Lastoria S, Yoshida WB, Rollo HA. Doenças vasculares periféricas. $3^{\text {a }}$ ed. Rio de Janeiro: MEDSI; 2002.

21. Moraes L, Puech-Leão LE, Toledo OM. Varizes e telangiectasias. Utilidade da microangiografia na orientação terapêutica. Rev Ass Med Bra. 1962;1:5-9.

22. Mello NA. Síndrome das Varizes. In: Melo NA. Síndromes vasculares. São Paulo: Byk; 1999. p. 265-94.

23. Thulesius O, Said S, Shuhaiber H, Neglen P, Gjores JE. Endothelial mediated enhancement of noradrenaline induced vasoconstriction in normal and varicose veins. Clin Physiol. 1991;11:153-9.

24. Rose SS, Ahmed A. Some thoughts on the aetiology of varicose veins. J Cardiovasc Surg (Torino). 1986;27:534-43.

25. Pitta GBB, Castro AA, Burihan E, editores. Angiologia e cirurgia vascular: guia ilustrado. Maceió: UNCISAL/ ECMAL; 2003. Disponível em: http://www.lava.med.br/livro.

26. Ungethuem ULG. Escleroterapia de varizes de membros inferiores por retropunção usando oleato de ethanolamina diluída em glicose hipertônica - análise de 12.560 casos. Anais do XII Congresso Internacional de Medicina Estética; 2003.

27. Pinto-Ribeiro A. Varizes dos membros inferiores - 38 E. Escleroterapia de varizes. In: Maffei FH. Doenças vasculares periféricas. Rio de Janeiro: MEDSI; 1987.

28. Centers for Disease Control and Prevention. Epi Info's home page. Disponível em: http://www.cdc.gov/epiinfo/Epi6/ EI6dwni.htm. Acessado: 15/10/2006.

29. Dean AG. Epi Info ${ }^{\text {TM }}$ and Epi Map: current status and plans for Epi Info $^{\text {TM }}$ 2000. J Public Health Manag Pract. 1999;5:54-7.

30. Breu FX, Guggenbichler S. European Consensus Meeting on Foam Sclerotherapy, April, 4-6, 2003, Tegernsee, Germany. Dermatol Surg. 2004;30:709-17.

31. Beale RJ, Gough MJ. Treatment options for primary varicose veins--a review. Eur J Vasc Endovasc Surg. 2005;30:83-95.

32. Rabe E, Pannier-Fischer F, Gerlach H, et al. Guidelines for sclerotherapy of varicose veins (ICD 10: I83.0, I83.1, I83.2, and I83,9). Dermatol Surg. 2004;30:687-93.

33. Correia ME, Oliveira AP. Complicações em escleroterapia. In: Pitta GBB, Castro AA, Burihan E, editores. Angiologia e cirurgia vascular: guia ilustrado. Maceió: UNCISAL/ ECMAL; 2003. Disponível em: http://www.lava.med.br/livro. 
34. Green RM. An update on the diagnosis and treatment of varicose veins. Prim Care Update Ob/Gyns. 1995;2:107-10.

35. Sparey C, Haddad N, Sissons G, Rosser S, de Cossart L. The effect of pregnancy on the lower-limb venous system of woman with varicose veins. Eur J Vasc Endovasc Surg. 1999;18:294-9.

36. Golledge J, Quigley FG. Pathogenesis of varicose veins. Eur J Vasc Endovasc Surg. 2003;25:319-24.

37. Jiang P, van Rij AM, Christie RA, Hill GB, Thomson IA. Venous physiology in the different patterns of recurrent varicose veins and the relationship to clinical severity. Cardiovasc Surg. 2000;8:130-6.
38. Psaila J, Melhuish J. Viscoelastic properties and collagen content of the long saphenous vein in normal and varicose veins. Br J Surg. 1989;76:37-40.

Correspondência:

Raimundo Rosendo de Oliveira

Av. Dix Sept Rosado, sn,

Edifício Dix Sept Rosado

CEP 59600-050 - Mossoró, RN

Tel.: (84) 9953.9961

E-mail: rosendo@mikrocenter.com.br 